\title{
Investigating the Reliability of HbA1c Monitoring for Blood Glucose Control During Late Pregnancy in Patients with Gestational Diabetes Mellitus (GDM) with and without $\beta$-Thalassemia Minor
}

\author{
Xueqin Zhang $\cdot$ Yunshan Xiao (D) - Yanfeng Fan
}

Received: July 17, 2018 / Published online: October 4, 2018

(C) The Author(s) 2018

\begin{abstract}
Introduction: Patients with gestational diabetes mellitus (GDM) need strict blood glucose control to reduce the incidence of perinatal complications in the mother or infant. The purpose of this study was to investigate whether the glycated hemoglobin (HbA1c) values of GDM patients were affected by $\beta$-thalassemia minor and to subsequently discuss the limitations of HbA1c monitoring for blood glucose control.

Methods: 41 GDM patients with $\beta$-thalassemia minor were enrolled to serve as the study group. 93 GDM patients without thalassemia were randomly selected as a control group. Clinical data on the 134 mothers as well as their newborns were retrospectively analyzed. The blood glucose values of the participants at various times during the gestation period were compared between the groups, as were their HbA1c
\end{abstract}

Xueqin Zhang, Yunshan Xiao, and Yanfeng Fan contributed equally to this work and should be considered co-first authors.

Enhanced Digital Features To view enhanced digital features for this article go to https://doi.org/10.6084/ m9.figshare.7093793.

X. Zhang · Y. Xiao $(\varangle) \cdot$ Y. Fan

Department of Obstetrics, Maternal and Child

Healthcare Hospital of Xiamen City, Xiamen,

Fujian, China

e-mail: xyssfp@163.com and ferritin levels and iron deficiency rates in late pregnancy (36-38 weeks of gestation). Pearson's coefficient was calculated to determine the correlations between HbA1c and ferritin in both the study and control groups.

Results: The study and control groups did not show any significant differences in newborn birth weight, maternal age, maternal pre-pregnancy body mass index (BMI), gestational age, newborn sex, gravidity, and parity. The blood glucose values of the participants at different times during the gestation period also did not differ significantly between the study group and the control group. However, the late-pregnancy HbA1c level (5.23 $\pm 0.49 \%)$ and iron deficiency rate $(12.19 \%)$ in the study group were significantly lower than those in the control group $(5.42 \pm 0.43 \%$ and $58.06 \%$, respectively); $P$ $<0.05$. Also, the late-pregnancy ferritin level in the study group $(46.59 \pm 18.03 \mathrm{ng} / \mathrm{mL})$ was significantly higher than that in the control group $(25.58 \pm 11.42 \mathrm{ng} / \mathrm{mL}) ; \quad P<0.05$. In addition, a significant negative correlation was observed between HbA1c and ferritin in both the study group $(R=-0.459, P=0.003)$ and the control group $(R=-0.358, P=0.010)$.

Conclusions: The HbA1c level is affected by many factors. Using serum HbA1c values to monitor blood glucose in GDM patients with $\beta$ thalassemia minor may lead to a mistaken assumption of low blood glucose levels, so HbA1c may not be a suitable indicator for monitoring blood glucose in pregnant women, 
particularly GDM patients with $\beta$-thalassemia minor.

Keywords: $\beta$-Thalassemia minor; Gestational diabetes mellitus; HbA1c

\section{INTRODUCTION}

Gestational diabetes mellitus (GDM), defined as hyperglycemia that is first discovered during pregnancy, is a high-risk condition for both mother and fetus. It is associated with adverse pregnancy outcomes, such as macrosomia (birth weight $>4000 \mathrm{~g}$ ), shoulder dystocia, stillbirth, and neonatal complications, including hypoglycemia and respiratory distress syndrome [1]. The long-term adverse effects of GDM on the metabolism and cardiovascular systems of mothers and their children pose a significant threat to public health [2].

Patients with GDM require strict blood glucose control to reduce the incidence of perinatal complications in the mother or the infant. Glycated hemoglobin (HbA1c) is widely accepted to be the most reliable indicator of longterm glycemic control [3]. HbA1c is generated by the nonenzymatic binding of glucose to the $\mathrm{N}$-terminal valine residue in a $\beta$ chain of hemoglobin. The HbA1c level in an individual's blood is proportional to the mean plasma glucose level over the lifespan of red blood cells (120 days) [4]. The Diabetes Control and Complications Trial provided extensive data on how the development and progression of complications depends on the HbA1c level in patients with diabetes mellitus [5].

HbA1c has also been used as an indicator of the blood glucose level in patients with GDM [6], but there is not enough clinical evidence to support its use in this context. Some studies have shown that HbA1c is affected by a variety of factors and thus cannot be used to accurately monitor blood glucose control during pregnancy [7]. HbA1c is a stable micro hemoglobin component that forms by the irreversible nonenzymatic reaction of glucose and hemoglobin in red blood cells [8]. The HbA1c concentration depends on plasma glucose levels and the RBC lifespan [9]. For example, it is known that the presence of hemoglobinopathy affects the results of almost any method of determining glycosylated hemoglobin [10].

The $\beta$-thalassemias are a group of hereditary hemoglobinopathies. They are caused by decreased or deficient synthesis of the $\beta$ chains of hemoglobin, and result in different phenotypes ranging from severely anemic to asymptomatic individuals. The incidence of this disease is very high in southern China [11]. In southern Guangdong and Guangxi, 2.54\% and $6.78 \%$ of the population are $\beta$-thalassemia carriers [12]. The severity of the thalassemia depends on the nature of the mutation. $\beta$-Thalassemia minor is caused by a mutation in one of two $\beta$-globin genes; this mutation does not severely damage the production of $\beta$ chains of hemoglobin, so the patient may be relatively asymptomatic. A review of the literature indicates that it is currently unclear how to evaluate HbA1c in GDM patients with $\beta$-thalassemia minor. The purpose of the work performed in the present study was to investigate whether the HbA1c levels of GDM patients are affected by $\beta$ thalassemia minor and to subsequently discuss the limitations of HbA1c monitoring for blood glucose control.

\section{METHODS}

\section{Study Population and Eligibility Criteria}

This study was a case-control study of women pregnant with singletons who underwent both antenatal examinations and delivery at the Department of Obstetrics of the Maternal and Child Healthcare Hospital of Xiamen, China, from July 2016 to December 2017. A total of 134 GMD patients were recruited for this study. In total, 41 GDM patients with $\beta$-thalassemia minor were enrolled to serve as the study group. Based on a study:control patient ratio of 1:2, 93 GDM patients without thalassemia were randomly selected as the control group. The inclusion criteria of the patients were as follows: (1) the mothers were diagnosed with GDM during gestational weeks 24-28; (2) singleton gestation; (3) complete information on both the mother and the newborn was available. 
Women who met the following criteria were excluded from the study: (1) those with pregestational diseases (hypertension, nephropathy, thyroid dysfunction, and diabetes), chronic alcohol ingestion, kidney disease, or blood loss; (2) multiple gestations; (3) fetuses with other genetic or congenital malformations; (4) birth occurred at $<36$ gestational weeks.

GDM was diagnosed if any glucose values exceeded the standard cutoff levels (fasting, $5.1 \mathrm{mmol} / \mathrm{L} ; \quad 1 \mathrm{~h}, 10.0 \mathrm{mmol} / \mathrm{L} ; \quad$ and $2 \mathrm{~h}$, $8.50 \mathrm{mmol} / \mathrm{L}$ ) in a $75 \mathrm{~g}$ oral glucose tolerance test performed during gestational weeks $24-28$ [13].

All GDM patients were treated with dietary adjustment and exercise or lifestyle modification combined with insulin. GDM patients were instructed to monitor blood glucose four times daily, including their morning fasting plasma glucose (FPG) and three 1-h postprandial glucose (PPG) values. Mean FPG (based on three values) and mean PPG (based on nine values) were calculated based on measurements performed on 3 days during the 32nd and 36th weeks of gestation, respectively, and the following glucose targets were aimed for: FPG 3.3-5.0 $\mathrm{mmol} / \mathrm{L}$ and 1 -h postprandial glucose $(\mathrm{PPG}) \leq 7.8 \mathrm{mmol} / \mathrm{L}$. Patients deficient in iron were advised to take iron supplements according to CSPM (Chinese Society of Perinatal Medicine) guidelines [14].

The inclusion criteria for the $\beta$-thalassemia minor group were as follows: only one of the two $\beta$-globin alleles contained a mutation, and there were no deletions or point mutations of the $\alpha$-globin gene.

All procedures performed in studies involving human participants were in accordance with the local ethics committee of the Maternal and Child Healthcare Hospital of Xiamen City (ky-2018-028) and with the 1964 Helsinki Declaration and its later amendments or comparable ethical standards. Informed written consent was obtained from each patient before their data were analyzed according to the requirements of the local ethics committee.

\section{Data Collection}

We recorded the following data: maternal age, pre-pregnancy body mass index (BMI), gravidity, parity, and family history of diabetes and hypertension. The number of gestational weeks was calculated based on the date of the last menstrual period, and was confirmed by ultrasound results. Data on neonatal outcomes were collected at the time of delivery. All of these data were entered into an electronic database.

All samples were collected in EDTA anticoagulant. The CBC was performed with an Advia 2120 analyzer (Siemens Medical Solutions Diagnostics, Tarrytown, NY, USA). Hemoglobin electrophoresis was carried out using a Capillarys 2 Flex Piercing analyzer (SEBIA Inc., Norcross, GA, USA) within one week after the blood sample was taken. Blood glucose levels were measured using a cobas 8000 series automatic biochemical analyzer (Roche Diagnostics, RischRotkreuz, Switzerland) at gestational weeks $8-10,20-24,30-32$, and 36-38. Ferritin was measured at gestational weeks 36-38 using chemiluminescence immunoassay (Advia Centaur, Siemens Medical Solutions Diagnostics). The HbA1c level was measured with a D10 fully automated glycosylated hemoglobin analyzer (Bio-Rad, Hercules, CA, USA) at gestational weeks 36-38.

\section{Globin Gene Analysis}

Screening for thalassemia in the pregnant women involved searching for patients with $\mathrm{MCV}<79.45 \mathrm{fL}, \mathrm{MCH}<25.15 \mathrm{pg}$, and $\mathrm{HbA} 2<$ $2.45 \%$ or $>3.75 \%$. Twenty-three mutations of the $\alpha$ - and $\beta$-globin genes were identified by a liquid-chip array system (model 200, Luminex Corp., Austin, TX, USA); see [15] for more on the methods used to screen for thalassemia and genotyping analysis.

\section{Statistical Analysis}

The data were analyzed using SPSS version 20.0. Data for normally distributed continuous variables are presented as mean $\pm \mathrm{SD}$ values. The $t$ test was applied to compare group means. 
Values of categorical variables are presented as frequencies. Differences between the two groups were checked for statistical significance using the chi-squared test. Pearson's coefficient was calculated to determine the correlation between two variables. A $p$ value of $<0.05$ was considered statistically significant.

\section{RESULTS}

The 134 participants in the study included 41 GDM patients with $\beta$-thalassemia minor (the study group) and 93 GDM patients without thalassemia (the control group). The clinical characteristics of the mothers and their newborns are summarized in Table 1 . There was no statistically significant difference between the groups in newborn birth weight, maternal age, maternal prepregnancy BMI, gestational age, newborn sex, gravidity, and parity $(P>0.05$ for each comparison; Table 1).
The blood glucose values of the participants in the study and control groups were measured at particular times during the gestation period. When the blood glucose values of the participants in the two groups were compared at each time point during the gestation period, there was no significant difference in the blood glucose values of the two groups $(p>0.05$ for each comparison, i.e., at each time point; Table 2).

The HbA1c levels in the study group were significantly lower than those in the controls (mean $\pm \mathrm{SD}=5.23 \pm 0.49 \%$ and $5.42 \pm 0.43 \%$ in the study and control groups, respectively; $P<0.05$ ).

The rate of iron deficiency in the study group $(12.19 \%)$ was significantly lower than that in the control group (58.06\%); $P<0.05$. The mean ferritin level in the study group $(46.59 \pm 18.03$ $\mathrm{ng} / \mathrm{mL}$ ) was significantly higher than that in the control group $(25.58 \pm 11.42 \mathrm{ng} / \mathrm{mL}) ; P<0.05$ (Table 3). Also, a significant negative correlation was observed between $\mathrm{HbA1c}$ and ferritin in

Table 1 Clinical characteristics of the 134 mothers and their newborns

\begin{tabular}{llll}
\hline & Study group $(\boldsymbol{n}=\mathbf{4 1})$ & Control group $(\boldsymbol{n}=\mathbf{9 3})$ & $\boldsymbol{p}$ \\
\hline Newborn birth weight $(\mathrm{g})$ & $3239.39 \pm 424.75$ & $3313.35 \pm 426.0$ & 0.354 \\
Maternal age (years) & $32.46 \pm 5.51$ & $32.32 \pm 4.48$ & 0.625 \\
Maternal pre-pregnancy BMI $\left(\mathrm{kg} / \mathrm{m}^{2}\right)$ & $21.36 \pm 2.91$ & $22.42 \pm 2.97$ & 0.058 \\
Gestational age (weeks) & $39.24 \pm 1.03$ & $39.15 \pm 1.25$ & 0.678 \\
Newborn sex & & & 0.162 \\
Male & $18(43.90 \%)$ & $53(53.90 \%)$ & \\
Female & $23(56.10 \%)$ & $40(43.01 \%)$ & 0.638 \\
Maternal gravidity & $10(24.39 \%)$ & $25(26.88 \%)$ & \\
1 & $12(29.26 \%)$ & $25(26.88 \%)$ & $43(46.23 \%)$ \\
2 & $19(46.34 \%)$ & & 0.922 \\
$\geq 3$ & $27(65.85 \%)$ & $57(61.29 \%)$ & $36(38.70 \%)$ \\
\hline
\end{tabular}

Values of continuous variables are given as the mean \pm SD. The $t$-test was applied to compare group means. Values of categorical variables are given as frequencies. Differences between the two groups were tested for statistical significance using the chi-squared test 
Table 2 Blood glucose values $(\mathrm{mg} / \mathrm{dL})$ of the participants in the two groups at various times during the gestation period

\begin{tabular}{|c|c|c|c|}
\hline $\begin{array}{l}\text { Time point at } \\
\text { which blood } \\
\text { glucose } \\
\text { measurement } \\
\text { occurred }\end{array}$ & $\begin{array}{l}\text { Study group } \\
(n=41)\end{array}$ & $\begin{array}{l}\text { Control } \\
\text { group } \\
(n=93)\end{array}$ & $p$ \\
\hline $\begin{array}{l}\text { At } 8-10 \text { weeks of } \\
\text { gestation }\end{array}$ & $4.99 \pm 0.56$ & $4.95 \pm 0.46$ & 0.607 \\
\hline \multicolumn{4}{|c|}{ At 24-28 weeks of gestation (OGTT) } \\
\hline Fasting & $4.78 \pm 0.41$ & $4.89 \pm 0.49$ & 0.239 \\
\hline $1 \mathrm{~h}$ postprandial & $10.05 \pm 1.48$ & $9.99 \pm 1.54$ & 0.859 \\
\hline \multicolumn{4}{|l|}{$2 \mathrm{~h}$ postprandial } \\
\hline $1 \mathrm{~h}$ postprandial & $8.43 \pm 1.72$ & $8.46 \pm 1.57$ & 0.902 \\
\hline \multicolumn{4}{|l|}{$2 \mathrm{~h}$ postprandial } \\
\hline $\begin{array}{l}\text { At } 30-32 \text { weeks of } \\
\text { gestation }\end{array}$ & $4.79 \pm 0.38$ & $4.86 \pm 0.64$ & 0.688 \\
\hline $\begin{array}{l}\text { At } 36-38 \text { weeks of } \\
\text { gestation }\end{array}$ & $4.64 \pm 0.99$ & $4.87 \pm 1.22$ & 0.327 \\
\hline
\end{tabular}

Values are expressed as the mean $\pm S D$; the $t$-test was applied to compare group means

Table $3 \mathrm{HbA1c}$ and ferritin levels and iron deficiency rates in late pregnancy (36-38 weeks of gestation) in the two groups

\begin{tabular}{llll}
\hline & $\begin{array}{l}\text { Study group } \\
(\boldsymbol{n}=\mathbf{4 1})\end{array}$ & $\begin{array}{l}\text { Control group } \\
(\boldsymbol{n}=\mathbf{9 3})\end{array}$ & $\boldsymbol{p}$ \\
\hline HbAlc (\%) & $5.23 \pm 0.49$ & $5.42 \pm 0.43$ & 0.027 \\
$\begin{array}{l}\text { Ferritin (ng/ } \\
\mathrm{mL})\end{array}$ & $46.59 \pm 18.03$ & $25.58 \pm 11.42$ & 0.001 \\
$\begin{array}{l}\text { Iron deficiency } \\
\text { rate }(\%)\end{array}$ & $12.19 \%(5 / 41)$ & $58.06 \%(39 / 93)$ & 0.035 \\
\hline
\end{tabular}

The values of HbAlc and ferritin shown are the mean $\pm \mathrm{SD}$, and the $t$-test was applied to compare group means. Data for the iron deficiency rate are shown as frequencies. Differences between the two groups were tested using the chi-squared test. A serum ferritin level of below $20 \mathrm{ng} / \mathrm{mL}$ was considered to indicate iron deficiency [14]
Table 4 Correlation between HbAlc and ferritin in the participants during late pregnancy (36-38 weeks of gestation)

\begin{tabular}{llll}
\hline Group & $\boldsymbol{n}$ & $\boldsymbol{R}$ & $\boldsymbol{p}$ \\
\hline Control & 93 & -0.358 & 0.010 \\
Study & 41 & -0.459 & 0.003 \\
\hline
\end{tabular}

Pearson's coefficient was calculated to determine the correlation between the two variables

A $p$ value of $<0.05$ was considered to indicate statistical significance

both the study group $(R=-0.459, P=0.003)$ and the control group $(R=-0.358, P=0.010)$; see Table 4.

\section{DISCUSSION}

Gestational diabetes mellitus (GDM), which has potentially adverse consequences, is a growing public health problem [16]. The prevalence of GDM in China has increased significantly in recent years [17]. Glucose control is the key medical intervention in cases of GDM.

The goal of glycemic control during pregnancy should be to keep blood sugar levels as close to normal as possible without inducing hypoglycemia. It is essential to find an accurate indicator of blood sugar, as this can provide valuable information for achieving glycemic control. Strict glycemic control should be implemented through self-monitoring of blood glucose (SMBG) or continuous glucose monitoring (CGM). It is difficult to perform blood glucose measurements in every patient, but blood glucose control can be monitored by measuring HbA1c. This approach is more specific and has lower biological and analytical variability than the fasting glucose test. There is a continuously increasing demand for the routine testing of HbA1c to detect diabetes mellitus and (especially) for long-term glycemic control [18].

Although the HbA1c reference interval for the general population is well established, the reference interval for healthy pregnant women is not clearly defined [19]. Different studies have 
reported different trends in HbA1c during pregnancy [20-22]: HbA1c levels during pregnancy can gradually or very frequently increase or decrease. It is difficult to use HbA1c to monitor glycemic control in GDM patients due to the dramatic changes in physical condition that occur during pregnancy [23] and the different genetic backgrounds of pregnant women. Thalassemia is an inherited hemoglobin disease. Hemoglobinopathy can affect the HbA1c value, potentially leading to an inaccurate judgment of the patient's blood glucose control [10].

The clinical manifestations of thalassemia are very diverse. The clinical classification scheme for $\beta$-thalassemia includes $\beta$-thalassemia major (TM; severe), $\beta$-thalassemia intermedia (TI; intermediate), and $\beta$-thalassemia minor (mild). TM refers to patients with severe anemia from the early stages of life on who require lifelong blood transfusion and iron chelation. TI patients are a highly diverse group who present various clinical severities: mild, moderate, and moderate-severe anemia. However, $\beta$-thalassemia minor is often asymptomatic or presents as mild anemia, and such patients do not require blood transfusion. Genetic counseling is needed. $\beta$-Thalassemia minor is caused by the presence of a single $\beta$ thalassemia mutation; the $\beta$-globin gene on the other chromosome is normal.

$\mathrm{HbA1c}$ is an indicator of hemoglobin glycosylation. The HbA1c concentration depends on the plasma glucose level and the lifespan of erythrocytes. Any condition that alters the lifespan of red blood cells may lead to an erroneous judgment of the HbA1c results [24]. It has been reported that the RBC lifespan is reduced in the presence of some variants of hemoglobin and in patients with thalassemia [25]. Therefore, the clinical significance of HbA1c in thalassemia patients may be misinterpreted.

This study found that GDM patients with $\beta$ thalassemia minor (the study group) showed no difference in blood glucose values from GDM patients without thalassemia (the control group) at various times during the gestation period, but that the HbA1c values of the study group were lower than those of the control group in late pregnancy. $\beta$-Thalassemia is an interfering factor when attempting to evaluate the level of HbA1c in GDM patients. This may be because $\beta$-thalassemia minor is potentially a hemolytic disease. We speculate that immature red blood cells are compensatory in $\beta$-thalassemia. The glycosylated hemoglobin of immature red blood cells is lower than that of mature red blood cells, causing HbA1c to decrease.

The HbA1c value of GDM patients with $\beta$ thalassemia minor is lower than that of GDM patients without thalassemia. This may be due to the iron load. When there is iron deficiency without blood loss, the lifespan of red blood cells is prolonged, increasing the saccharification reaction time and increasing the level of glycosylated hemoglobin [26]. This study found that there was a negative correlation between serum ferritin and HbA1c in both the study group and the control group. Compared with the control group, the incidence of iron deficiency in the study group was significantly lower, while the serum ferritin content was significantly higher. However, iron overload was not observed in the study group.

Iron demand increases during pregnancy. Iron deficiency is a common phenomenon among pregnant women, especially those in late pregnancy [27]. The same is true of GDM patients. In our study, the incidence of iron deficiency in GDM patients without thalassemia was $58.06 \%$ in late pregnancy. Studies have shown that normal pregnant women and diabetic patients have higher HbA1c levels in the third trimester [21]. Most GDM patients benefit from blood glucose control. The level of fasting blood glucose (FBG) tends to decrease during pregnancy. Thus, if HbA1c was only a reflection of the average blood sugar level, it would decrease as pregnancy progresses. This suggests that other factors besides the blood glucose level can affect the HbA1c value. This interference may be due to the increased likelihood of iron deficiency late in pregnancy.

$\beta$-Thalassemia minor may inhibit the level of serum hepcidin [28]. Hepcidin is considered to be a key regulator of the iron balance [29]. It is a negative regulator of the absorption of iron from the intestine and the release of iron from macrophages. Suppressing hepcidin can enhance iron absorption. Patients with $\beta$ - 
thalassemia minor characteristically present a slow-moving increase in iron loading [28]. There may be hitherto unrecognized mechanisms for more effectively adapting to chronic anemia. In this study, the incidence of iron deficiency in GDM patients with $\beta$-thalassemia minor was only $12.19 \%$ during late pregnancy.

In summary, thalassemia, a hemolytic disease, leads to a shortened hemoglobin lifespan. Any condition that alters the lifespan of red blood cells may influence the HbA1c value. Moreover, thalassemia promotes iron load levels in pregnant women, and iron load is negatively correlated with $\mathrm{HbA1c}$. Both of these causes led to lower levels of HbA1c in GDM patients with $\beta$-thalassemia minor than in those without thalassemia.

Maybe we should consider modifying the reference interval for HbA1c according to factors such as gestational age, ferritin level, hemoglobin level, and whether or not thalassemia is present. However, this would be very difficult because there are so many factors that affect the HbA1c value during pregnancy. Since the reference interval would therefore be rather complicated to determine, it would be hard to apply it clinically. While we continue to search for a more appropriate indicator of blood glucose level than HbA1c, in order to avoid misjudgments, we must approach clinical test results and literature reports based on HbA1c data with caution when they relate to pregnant patients, and we must identify all of the factors that interfere with the determination of HbA1c.

To properly manage GDM, it is critical to find suitable indicators for monitoring blood glucose control. At present, HbA1c is widely used for monitoring blood glucose control and for diabetes diagnosis. However, the HbA1c level is affected by many factors. Serum HbA1c values in GDM patients with $\beta$-thalassemia minor may suggest misleadingly low blood glucose levels. Therefore, care should be taken when using HbA1c for glycemic control assessment. We need to find more reliable indicators.

There are several limitations of this study. First, the HbA1c values of the GDM patients with $\beta$-thalassemia minor may have been influenced by factors not considered in this study. More detailed investigations of this issue are needed, such as prospective clinical trials and studies using animal models. Second, more cases and more controls are needed to examine this topic in more depth; for example, to explore the degree to which $\mathrm{HbA1c}$ is underestimated in patients with $\beta$-thalassemia minor and poorly controlled GDM. In this way, the limitations of HbA1c monitoring for blood glucose control can be probed in detail.

\section{CONCLUSIONS}

The HbA1c level is affected by various factors during pregnancy, making it difficult to establish a reference interval for pregnant women. Thalassemia is an inherited hemoglobin disease, and hemoglobinopathy can result in misleadingly low $\mathrm{HbA1c}$ values. Therefore, HbA1c is not a suitable indicator for monitoring blood glucose control in pregnant women, especially GDM patients with $\beta$-thalassemia minor.

\section{ACKNOWLEDGEMENTS}

We thank the participants of the study.

Funding. No funding or sponsorship was received for this study or the publication of this article. The article processing charges were funded by the authors.

Authorship. All named authors meet the International Committee of Medical Journal Editors (ICMJE) criteria for authorship for this article, take responsibility for the integrity of the work as a whole, and have given their approval for this version to be published.

Authorship Contributions. Xueqin Zhang designed this study and edited the manuscript. Yunshan Xiao collected data and wrote the manuscript. Yanfeng Fan performed the statistical analysis. These authors contributed equally to this work.

Disclosures. Xueqin Zhang, Yunshan Xiao, and Yanfeng Fan have nothing to disclose. 
Compliance with Ethics Guidelines. All procedures performed in studies involving human participants were in accordance with the local ethics committee of the Maternal and Child Healthcare Hospital of Xiamen City (ky2018-028) and with the 1964 Helsinki Declaration and its later amendments or comparable ethical standards. Informed consent was obtained from all individuals included in the study.

Data Availability. The data sets generated and analyzed during the current study are available from the corresponding author on reasonable request.

Open Access. This article is distributed under the terms of the Creative Commons Attribution-NonCommercial 4.0 International License (http://creativecommons.org/licenses/ by-nc/4.0/), which permits any noncommercial use, distribution, and reproduction in any medium, provided you give appropriate credit to the original author(s) and the source, provide a link to the Creative Commons license, and indicate if changes were made.

\section{REFERENCES}

1. Metzger BE, Lowe LP, Dyer AR, Trimble ER, Chaovarindr U, Coustan DR, Hadden DR, McCance DR, Hod M, McIntyre HD. Hyperglycemia and adverse pregnancy outcomes. N Engl J Med. 2008;358:1991-2002.

2. Barbour LA. Changing perspectives in pre-existing diabetes and obesity in pregnancy: maternal and infant short- and long-term outcomes. Curr Opin Endocrinol Diabetes Obes. 2014;21:257-63.

3. World Health Organization. Use of glycated hemoglobin (HbA1c) in the diagnosis of diabetes mellitus: abbreviated report of WHO consultation. Geneva: World Health Organization; 2011.

4. Tahara Y, Shima K. Kinetics of HbA1c, glycated albumin, and fructosamine and analysis of their weight functions against preceding plasma glucose level. Diabetes Care. 1995;18:440-7.

5. The Diabetes Control and Complications Trial Research Group. The effect of intensive treatment of diabetes on the development and progression of long-term complications in insulin-dependent diabetes mellitus. N Engl J Med. 1993;329:977-86. https://doi.org/10.1056/NEJM199309303291401.

6. Blumer I, Hadar E, Hadden DR, Jovanovič L, Mestman JH, Murad MH, Yogev Y. Diabetes and pregnancy: an Endocrine Society clinical practice guideline. $\mathrm{J}$ Clin Endocrinol Metab. 2013;98:4227-49. https://doi.org/10.1210/jc.20132465.

7. Hughes RCE, Rowan J, Florkowski CM. Is there a role for HbA1c in pregnancy? Curr Diab Rep. 2016;16:5.

8. Bunn HF, Haney DN, Kamin S, Gabbay KH, Gallop PM. The biosynthesis of human hemoglobin A1c. J Clin Invest. 1976;57:1652-9.

9. Panzer S, Kronik G, Lechner K, Bettelheim P, Neumann E, Dudczak R. Glycosylated hemoglobin $(\mathrm{GHb})$ : an index of red cell survival. Blood. 1982;59:1348-50.

10. Ji L, Yu J, Zhou Y, Xia Y, Xu A, Li W, et al. Erroneous HbA1c measurements in the presence of $\beta$-thalassemia and common Chinese hemoglobin variants. Clin Chem Lab Med. 2015;53:1451-8. https:// doi.org/10.1515/cclm-2014-0598.

11. Li CG, Li CF, Li Q, Li M. Thalassemia incidence and treatment in China with special reference to Shenzhen City and Guangdong Province. Hemoglobin. 2009;33(5):296-303.

12. Chen W, Zhang X, Shang X, Cai R, Li L, Zhou T, et al. The molecular basis of beta-thalassemia intermedia in southern China: genotypic heterogeneity and phenotypic diversity. BMC Med Genet. 2010;11:31.

13. World Health Organization. Diagnostic criteria and classification of hyperglycaemia first detected in pregnancy. A World Health Organization guideline. Diabetes Res Clin Pract. 2014;103:341-63. https:// doi.org/10.1016/j.diabres.2013.10.012.

14. Chinese Society of Perinatal Medicine. Guideline on iron deficiency and iron deficiency anemia in pregnant women and premenopausal women. Chin J Perinat Med. 2014;14(7):451-4.

15. Yin A, Li B, Luo $M$, et al. The prevalence and molecular spectrum of alpha- and beta-globin gene mutations in 14,332 families of Guangdong Province, China. PLoS One. 2014;9:e89855. https:// doi.org/10.1371/journal.pone.008985515.

16. Carroll X, Liang X, Zhang W, Zhang W, Liu G, Turner N, Leeper-Woodford S. Socioeconomic, environmental and lifestyle factors associated with gestational diabetes mellitus: a matched case-control 
study in Beijing, China. Sci Rep. 2018;8(1):8103. https://doi.org/10.1038/s41598-018-26412-6.

17. Tutino GE, Tam WH, Yang $\mathrm{X}$, et al. Diabetes and pregnancy: perspectives from Asia. Diabetic Med. 2014;31:302-18.

18. Nathan DM, Balkau B, Bonora E, Borch-Johnsen K, Buse JB, Colagiuri $S$, et al. International Expert Committee report on the role of the A1c assay in the diagnosis of diabetes. Diabetes Care. 2009;32:1327-34.

19. Buchanan TA, Xiang AH, Page KA. Gestational diabetes mellitus: risks and management during and after pregnancy. Nat Rev Endocrinol. 2012;8(11):639-49.

20. Rafat D, Ahmad J. HbA1c in pregnancy. Diabetes Metab Syndr. 2012;6(1):59-64.

21. Hiramatsu Y, Shimizu I, Omori Y, Nakabayashi M, JGA (Japan Glycated Albumin Study Group). Determination of reference intervals of glycated albumin and hemoglobin A1c in healthy pregnant Japanese women and analysis of their time courses and influencing factors during pregnancy. Endocr J. 2012;59:145-51.

22. Nielsen LR, Ekbom P, Damm P, Gl€umer C, Jensen $\mathrm{DM}$, Frandsen MM, et al. HbA1c levels are significantly lower in early and late pregnancy. Diabetes Care. 2004;27:1200-1.

23. Carreiro MP, Nogueira AI, Ribeiro-Oliveira A. Controversies and advances in gestational diabetes-an update in the era of continuous glucose monitoring. J Clin Med. 2018:7(2):11. https://doi.org/10. 3390/jcm7020011.

24. NGSP. NGSP certified methods and laboratories. www.ngsp.org. Accessed 6 Jul 2018.

25. Wasserman M, Rubin H. True red blood cell survival with radiochromium by correcting for percentage of activity not bound to hemoglobin: demonstration of two erythrocyte populations in normals and in thalassemia minor: erythropoiesis and the thalassemia trait. Ann NY Acad Sci. 1964;119:586-606.

26. Christy AL, Manjrekar PA, Babu RP, Hegde A. Influence of iron deficiency anaemia on haemoglobin A1C levels in diabetic individuals with controlled plasma glucose levels. Iran Biomed J. 2014;18(2):8827.

27. Hashimoto K, Koga M. Influence of iron deficiency on HbA1c levels in pregnant women: comparison with non-pregnant women. J Clin Med. 2018;7(2):34. https://doi.org/10.3390/jcm7020034.

28. Jones E, Pasricha SR, Allen A, et al. Hepcidin is suppressed by erythropoiesis in hemoglobin $\mathrm{E} \beta$ thalassemia and $\beta$-thalassemia trait. Blood. $2015 ; 125(5): 873-80$.

29. Ganz T, Nemeth E. The hepcidin-ferroportin system as a therapeutic target in anemias and iron overload disorders. Hematol Am Soc Hematol Educ Progr. 2011;2011:538-42. 